\title{
Radiofrequency Excitation
}

\section{OVERVIEW}

The image, or measured spin density in an MRI experiment, is not necessarily equivalent to the physical spin density of the object being imaged, even when relaxation and Fourier transform effects are taken into account. The image is actually a picture of the signal received by the RF receive coil or RF probe. It is, in fact, proportional to the product of the field (which would be produced by the receive coil) and the transverse magnetization (which itself depends on the transmit coil's field). This has been discussed in UNIT B2.1.

When either the transmit or receive coil is not perfect, or when the slice select gradients exist, the flip angle becomes a function of space. These effects will take a uniform object and from it create a nonuniform image (see Fig. B2.2.1). In this unit, we focus on the effect from the slice select gradients. (More details of the slice select gradients are discussed in Chapter B4.) In order to excite a slice through the object or person being imaged, the slice select gradients and the RF field have to be on at the same time. The exact values of the flip angle and the magnetization can be solved from the Bloch equations. In most cases, the Bloch equations are solved numerically. Figure B2.2.2 plots magnetization in the transverse plane $\left(M_{\perp}\right)$ as a function of time with three different frequency offsets. When it is on-resonance, $M_{\perp}$ is proportional to the sine of the flip angle, with the solution presented in UNIT B1.2.

If the flip angle is small, then an analytical formula can be derived as an approximate solution of the Bloch equations. This analytical form of the magnetization gives us a rough idea as to how the signal behaves. When the spins are tipped into the transverse plane, because these spins are at different locations, they precess around the gradient field at different rates. The imaging signal is proportional to the sum of the transverse spins. Thus, if the spins have different phases, the signal will be low. If a gradient that has an opposite polarity of the slice select gradient is applied, these spins can be brought back so that they are aligned along the same direction. For low flip angles, the area under this extra gradient must be $50 \%$ of that under the original slice select gradient during which the RF is on. This is what we mean by refocusing or rephasing the spins. A simple sequence diagram demonstrating this is shown in Figure B2.2.3. With this refocusing gradient, Figure B2.2.4 shows that the $x$ component of the magnetization, $M_{x}$, is zero if the RF field is along the $x$-direction in the rotating frame. When the flip angle is not small, the refocusing gradient lobe is not exactly $50 \%$ of the area under the slice select gradient lobe. From a numerical solution of the Bloch equations, Figure B2.2.5 demonstrates that $M_{x}$ is not close to zero when the area of the refocusing gradient lobe is either $50 \%$ or $52 \%$ of the area under the slice select gradient. When the flip angle is $90^{\circ}$ and the refocusing area is $\sim 50.6 \%$ of the area under the slice select gradient, the spins are properly rephased.

The low flip angle solution also helps us to understand the RF field behavior in the frequency domain, which is the inverse Fourier transform of the RF field in the time domain. Because the flip angle is small, the RF field in the frequency domain is proportional to the flip angle (or the magnetization). Figures B2.2.6A and B2.2.6B are such Fourier transform duals. Because the RF field in Figure B2.2.6A is truncated, the Gibbs ringing appears in the flip angle profile shown in Figure B2.2.6B. We can apply a Hanning filter to the RF field (Fig. B2.2.6C), and the flip angle becomes uniform across the slice profile, although the filter causes the profile to be broadened (Fig. B2.2.6D).

Contributed by Yu-Chung Norman Cheng and E. Mark Haacke

Current Protocols in Magnetic Resonance Imaging (2004) B2.2.1-B2.2.11

Copyright () 2004 by John Wiley \& Sons, Inc.
Radiofrequency Excitation and Reception

B2.2.1

Supplement 12 
With a combination of the slice select gradients and the RF field, it is possible to excite a beam of spins. In Figure B2.2.7A, the $\pi / 2$-pulse and the $z$ slice select gradient excite a slice in the $x-y$ plane (see Fig. B2.2.7B). The $\pi$-pulse and the $y$ gradient excite a slice in the $x-z$ plane. This yields a beam of spins (Fig. B2.2.7B) which experience a spin echo. This sequence can be used to image small regions (fields-of-view) in the body most efficiently as it shortens the imaging time because less phase encoding steps are required.

\section{TECHNICAL DISCUSSION}

In UNIT B2.1, it was shown that the signal measured in an MRI experiment is proportional to the emf picked up by the RF receive coil which, in turn, is proportional to the scalar product of the transverse magnetization and the receive RF field (see Equation B2.1.10). We now try to understand how variations in the RF field will cause variations in image intensity that are independent of the physical spin density.

\section{A Brief Review of Signal}

The signal received from a differential volume element $d^{3} r$ is:

$$
d s(\vec{r}) \propto M_{\perp}(\vec{r}) \mathcal{B}_{\perp}^{\text {receive }}(\vec{r}) d^{3} r
$$

where the time dependence of the signal has been ignored. After Fourier transforming a frequency-encoded signal with respect to time, an image is produced which is proportional to the same terms:

$$
\hat{\rho}(\vec{r}) \propto M_{\perp}(\vec{r}) \mathcal{B}_{\perp}^{\text {receive }}(\vec{r})
$$

The reconstructed image is said to represent the physical spin density, although it is seen that other quantities are involved in this expression. Remember that $M_{\perp}(\vec{r})$ is usually generated from the creation of transverse magnetization when a set of spins is tipped by the RF pulse. To find the relationship between the measured spin density and the physical spin density, it is necessary to determine how $M_{\perp}(\vec{r})$ is related to $B_{1}{ }^{\text {transmit }}(\vec{r}, t)$ and the applied slice select gradients.

The magnitude of the perpendicular component of the magnetization, neglecting relaxation and assuming the equilibrium magnetization $M_{0}$ is available, is dependent on the flip angle, $\theta(\vec{r})$, at that position, via:

$$
M_{\perp}(\vec{r})=M_{0}(\vec{r}) \sin \theta(\vec{r})
$$

The spatial effects of the transmit $R F$ field and applied gradients are reflected in $\theta(\vec{r})$. It is generally assumed in imaging that $\theta(\vec{r})$ is constant over the excited region, but this is the case only if the RF field is perfectly uniform and perfect slice selection occurs. However, neither of these conditions exists in reality (see Fig. B2.2.1).

\section{Numerical Solutions of the Bloch Equations}

The exact values of $M_{\perp}(\vec{r})$ and $\theta(\vec{r})$ can be numerically obtained from the Bloch equations. From UNIT B1.2 the reader may recall the equation of motion of the magnetization in the rotating frame. In order to excite a slice, a slice select gradient $\vec{G}$ has to be turned on when the RF field is on. The slice select gradient leads to a fixed range of frequencies being excited that creates a slice whose normal $\vec{r}$ is parallel to $\vec{G}$. All spins inside the desired slice are then tipped from the $z$-axis by the RF pulse. With the presence of slice select gradients and an RF field $\vec{B}_{1}$ and neglecting relaxation times, the Bloch equation becomes: 

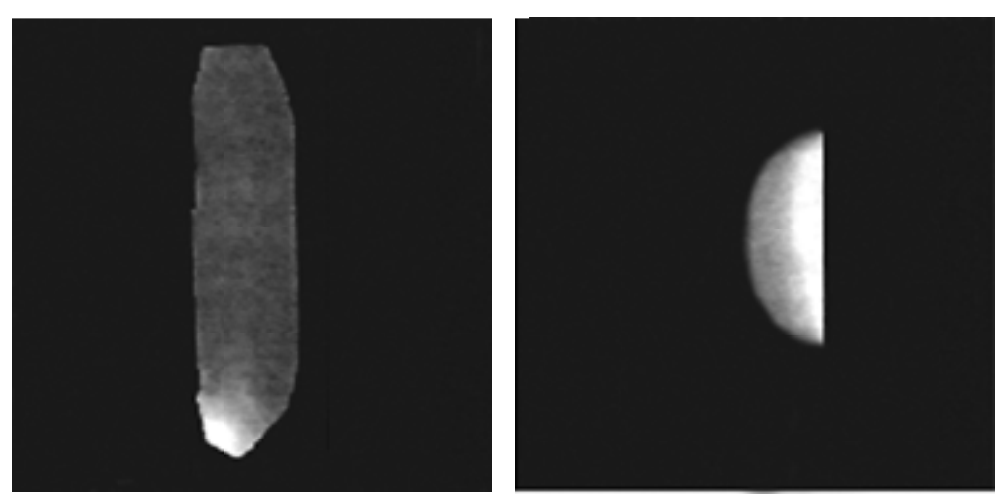

Figure B2.2.1 A uniform-spin-density object is placed in two different coils. (A) The first image is acquired with a bird-cage coil used for both transmission of the RF pulse and reception of the signal. The RF field $\left.B_{1}{ }^{\text {transmit }} \overrightarrow{(r}\right)$ produced by this bird-cage coil is approximately uniform over the object, and a fairly uniform image results. (B) The same bird-cage coil as in panel A was used to tip the spins, but a surface coil was used to receive the signal. The spatial dependence of $\mathrm{B}_{\perp}{ }^{\text {receive }}$ leads to a change in intensity of the image as a function of position across the image. Imaging parameters: (A) $T_{R} / T_{E}=500 \mathrm{~ms} / 12 \mathrm{~ms}, T_{s}=7.68 \mathrm{~ms}, \Delta \mathrm{x} \times \Delta \mathrm{y} \times \Delta \mathrm{z}=1.95 \mathrm{~mm} \times 1.95 \mathrm{~mm} \times 3 \mathrm{~mm}, N_{x} \times N_{y}=$ $256 \times 128, \theta=90^{\circ}, N_{a c q}=1, \tau_{\mathrm{RF}}=2.56 \mathrm{~ms}, G_{s s}=8 \mathrm{mT} / \mathrm{m}$; (B) $N_{a c q}=2$.

$$
\frac{d \vec{M}}{d t}=\gamma \vec{M} \times\left(\vec{B}_{1}+(\vec{G} \cdot \vec{r}) \hat{z}\right)
$$

If the components of the RF field lie in the $x$-y plane, i.e., $\vec{B}_{1}=\left(B_{1 x}, B_{1 y}, 0\right)$, Equation B2.2.4 can be rewritten as:

$$
\left(\begin{array}{c}
\frac{d M_{x}}{d t} \\
\frac{d M_{y}}{d t} \\
\frac{d M_{z}}{d t}
\end{array}\right)=\gamma\left(\begin{array}{rrr}
0 & \vec{G} \cdot \vec{r} & -B_{1 y} \\
-\vec{G} \cdot \vec{r} & 0 & B_{1 x} \\
B_{1 y} & -B_{1 x} & 0
\end{array}\right)\left(\begin{array}{l}
M_{x} \\
M_{y} \\
M_{z}
\end{array}\right)
$$

We can simulate the behavior of a set of spins from these equations. Figure B2.2.2 demonstrates the behavior of the transverse magnetization as a function of time during the RF pulse for spins on-resonance and off-resonance. The solid line in Figure B2.2.2 shows $M_{\perp}(t)$ during an applied sinc pulse for on-resonance spins. (The sinc function is defined later.) When on-resonance, the $\vec{G} \cdot \vec{r}$ term vanishes in the Bloch equation and the solution of $M_{\perp}$ is the simplified form given in UNIT B1.2. The dashed line in Figure B2.2.2 shows $M_{\perp}(t)$ during the same pulse for off-resonance spins near the edge of the slice (where we take the resonance offset as $\Delta f=\mathrm{BW}_{\mathrm{RF}} / 2$ and $\mathrm{BW}_{\mathrm{RF}}$ is the RF excitation bandwidth). An off-resonance condition with $\Delta f=\mathrm{BW}_{\mathrm{RF}}$ is also shown for comparison. The bandwidth is related to the slice select gradient $G_{\text {ss }}$ through $\vec{G} \cdot \vec{r}=G_{\mathrm{ss}} T H=$ $\mathrm{BW}_{\mathrm{RF}} / ₹$ where $T H$ is the slice thickness. Thus, the slice thickness is defined by the RF bandwidth and slice select gradients.

Radiofrequency Excitation and Reception

\section{B2.2.3}

Supplement 12 


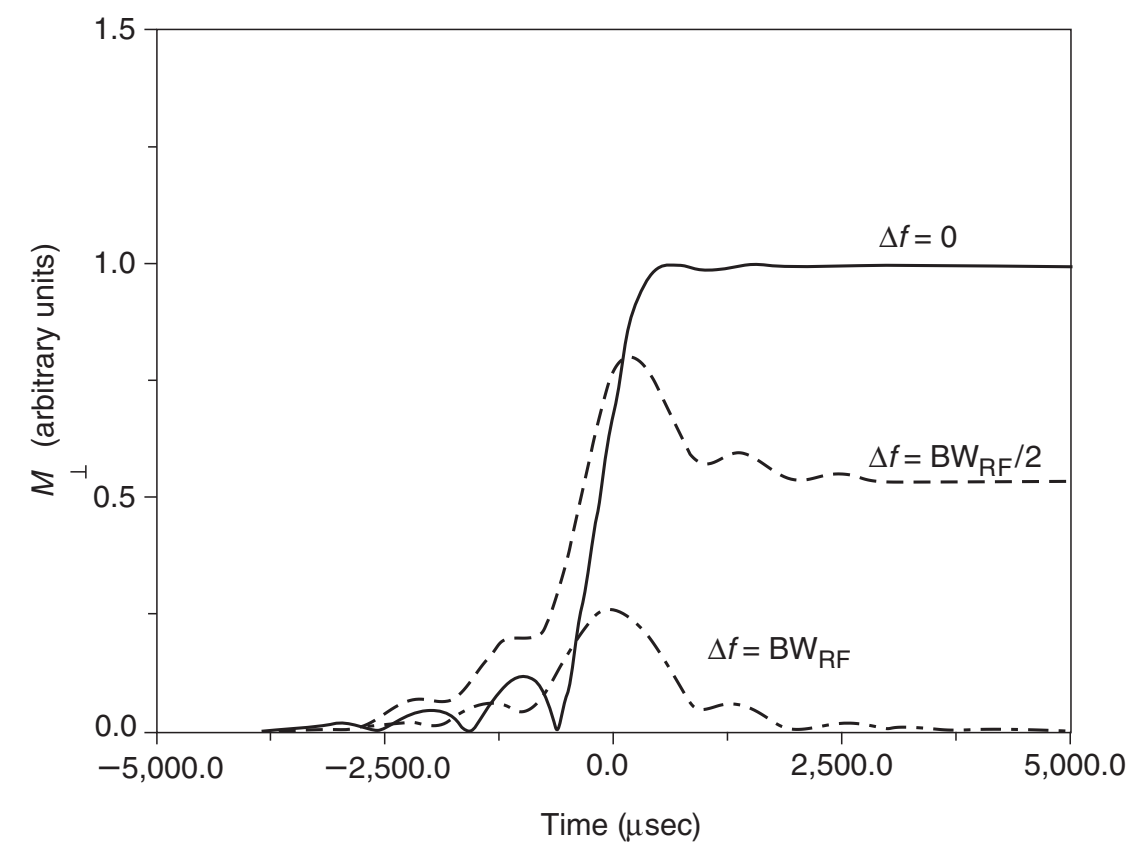

Figure B2.2.2 $M_{\perp}(t)$ during an applied sinc RF pulse for on-resonance and off-resonance spins. Here, $\Delta f$ is the frequency offset in $\mathrm{Hz}$ and $\mathrm{BW}_{\mathrm{RF}}$ is the RF excitation bandwidth.

\section{Dephasing and rephasing across a slice at low flip angles}

The arguments presented so far assume that the spins behave in a simple linear fashion so that, for example, if $B_{1}^{\text {transmit }}$ doubles, so does $\theta$. In practice, this only holds true when the spins are on-resonance and the direction of $B_{1}{ }^{\text {transmit }}$ is a time-independent vector in the rotating frame. This is also approximately correct when the flip angle is small. Consider a further approximation of Equation B2.2.5. If only small tip angles are considered such that $\cos \theta \cong 1$ and $M_{z} \cong M_{0}$, then the transverse and longitudinal equations can be decoupled. The resulting equations for $M_{x}$ and $M_{y}$ can be combined by choosing:

$$
M_{+} \equiv M_{x}+i M_{y}
$$

and

$$
B_{1} \equiv B_{1 x}+i B_{1 y}
$$

then the equation of motion reduces to:

$$
\frac{d M_{+}}{d t}=-i \gamma(\vec{G} \cdot \vec{r}) M_{+}+i \gamma B_{1} M_{0}
$$

where the explicit spatial dependence of $\vec{G}$ and $B_{1}$ are left as understood and $M_{z}$ has been replaced by $M_{0}$ in Equation B2.2.8.

A general solution can be used to illustrate the dephasing of the spins across the slice. Assume initial conditions of $M_{x}\left(-\tau_{\mathrm{RF}} / 2\right)=M_{y}\left(-\tau_{\mathrm{RF}} / 2\right)=0, M_{z}\left(-\tau_{\mathrm{RF}} / 2\right)=M_{0}$, and that $B_{1}(t)$ is nonzero over the interval $\left(-\tau_{\mathrm{RF}} / 2, \tau_{\mathrm{RF}} / 2\right)$. Using, for example, the integrating factor technique, the solution of Equation B2.2.8 at the end of the RF pulse, $t=\tau_{\mathrm{RF}} / 2$, is: 


$$
M_{+}\left(\vec{r}, \tau_{\mathrm{RF}} / 2\right)=i \gamma M_{0} \int_{-\tau_{\mathrm{RF}} / 2}^{\tau_{\mathrm{RF}} / 2} d t B_{1}(t) e^{-i 2 \pi \vec{k}(t) \cdot \vec{r}}
$$

where:

$$
\vec{k}(t)=\nsucc \int_{t}^{\tau_{\mathrm{RF}} / 2} d s \vec{G}(s)
$$

For $\vec{G}(s)=\vec{G}$, a constant:

$$
\vec{k}(t)=\gamma\left(\tau_{\mathrm{RF}} / 2-t\right) \vec{G}
$$

and:

$$
\begin{aligned}
M_{+}\left(\vec{r}, \tau_{\mathrm{RF}} / 2\right) & =i \gamma M_{0} e^{-i \gamma \vec{G} \cdot \vec{r} \tau_{\mathrm{RF}} / 2} \int_{-\tau_{\mathrm{RF}} / 2}^{\tau_{\mathrm{RF}} / 2} d t B_{1}(t) e^{i \gamma \vec{G} \cdot \vec{r} t} \\
& =i \gamma M_{0} e^{-i \gamma \vec{G} \cdot \vec{r} \tau_{\mathrm{RF}} / 2} \mathcal{F}^{-1}\left(B_{1}(t)\right)
\end{aligned}
$$

where $B_{1}(t)$ is taken to be zero outside the RF window.

If $B_{1 y}$ is assumed to be zero and $B_{1 x}$ is a real and symmetric function, the inverse Fourier transform of $B_{1}(t)$ will also be real and symmetric. The explicit phase term $e^{-i \gamma \vec{G} \cdot \vec{r}\left(\tau_{\mathrm{RF}} / 2\right)}$ in Equation B2.2.12 is a function of space and represents the dephasing across the slice during slice selection. (The reader may recall from UNIT B2.1 that the Fourier transform of $M_{\perp}$ is the signal rather than $M_{\perp}$ itself. Thus, this spatially dependent phase term should be eliminated in order to have a stronger signal. By eliminating this unwanted phase, we bring the spins to the same direction. This is called refocusing or rephasing.) Rephasing must be performed after the RF pulse is turned off to get an accurate description of the magnetization as it appears just prior to frequency encoding. A "rephasing gradient" of amplitude $G_{r p}$ is turned on after the RF pulse so that the phase it generates cancels the phase term $-\gamma \vec{G} \cdot \vec{r}\left(\tau_{\mathrm{RF}} / 2\right)$ in the above equation. Referring to Figure B2.2.3, the phase $\phi_{r p}$ generated at the end of the rephase gradient lobe of amplitude $G_{r p}$ must be:

$$
\phi_{r p}=\gamma G_{r p} \hat{z} \cdot \vec{r} \tau_{r p}=\gamma G_{r p} z \tau_{r p}
$$

such that $\phi_{r p}$ cancels the phase in Equation B2.2.12. This yields:

$$
\vec{G} \cdot \vec{r} \frac{\tau_{\mathrm{RF}}}{2}=G_{0, z} z \tau_{\mathrm{RF}} / 2=G_{r p} z \tau_{r p}
$$

If the area $G_{r p} \tau_{r p}$ satisfies Equation B2.2.14, it brings all spins back into phase along the $y$ direction, along which on-resonance spins are tipped. If we choose $G_{r p}=G_{0, z}$, then $\tau_{r p}$ $=\tau_{\mathrm{RF}} / 2$ will lead to refocusing. Intuitively, if one views the RF pulse as instantaneously exciting the spins at $t=0$, then the spins in the transverse plane only see $G_{0, z}$ for $\tau_{\mathrm{RF}} / 2$ and, therefore, only one-half of the area under $G_{0, z}$ will be needed to refocus the spins.

Radiofrequency Excitation and Reception

B2.2.5 


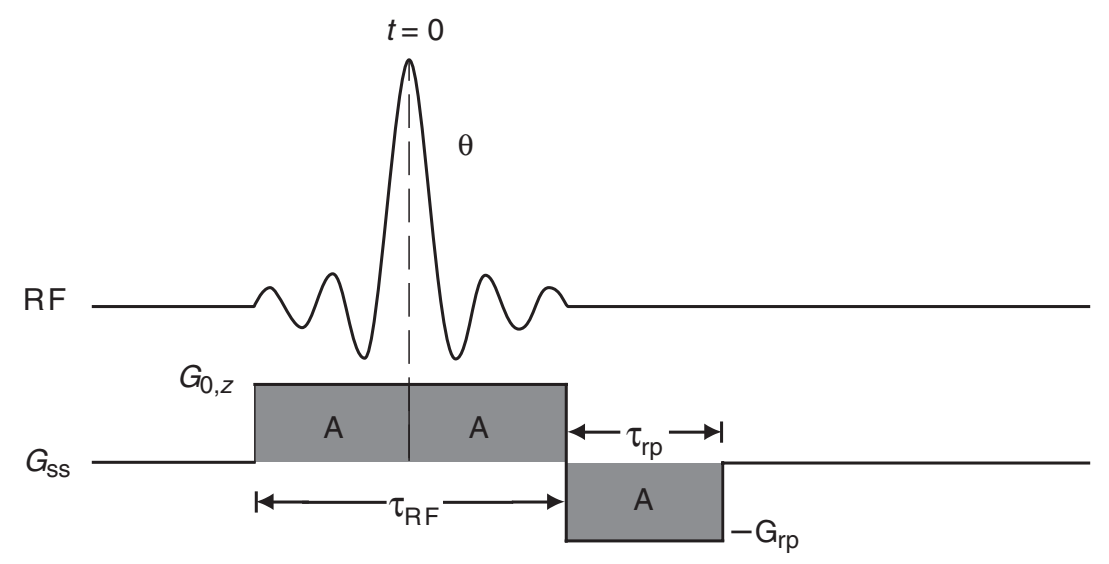

Figure B2.2.3 The RF pulse and slice select gradient waveforms (assumed to be along $\hat{z}$ ). Refocusing occurs thanks to the effect of the negative lobe with amplitude $G_{r p}$.

\section{Rephasing at large flip angles}

To find out what happens for large flip angles, the Bloch equations must be solved. In the examples to follow, a numerical method has been used to solve the Bloch equations during a slice select pulse at several flip angles. It is assumed that $B_{1}{ }^{\text {transmit }}$ is spatially homogeneous for these simulations and applied along the $\hat{x}$-axisintherotatingframe.

Figure B2.2.4 shows the magnetization as a function of $z$ for a low flip angle pulse which has been rephased with a gradient area equal to half of the slice select lobe. This result shows that the low flip angle approximation is accurate, since the $50 \%$ refocusing has eliminated any dephasing ( $M_{x}$ is zero), and the only remnant errors in the profile are due to truncation of the RF pulse. Panels A and B in Figure B2.2.5 show $\pi / 2$-pulse excitation profiles after refocusing with areas of $50 \%$ and $52 \%$, respectively. The presence of a nonzero $M_{x}$ component introduces a $z$-dependent phase behavior, if the slice select gradient is along the $z$-direction. The choice of $50.6 \%$ refocusing minimizes the $M_{x}$ profile. The need for a non $50 \%$ refocusing pulse is indicative of the nonlinearities of the Bloch equations. In order to determine the result of a large flip angle pulse, a numerical method should be employed. Note that in our simulations, a total of 16 zero crossings of a truncated RF sinc function were used [i.e., $B_{1} \operatorname{sinc}\left(\pi t / \tau_{\mathrm{zc}}\right)$, where $t$ is between $-8 \tau_{\mathrm{zc}}$ and $8 \tau_{\mathrm{zc}}$ ]. Also, the amplitude of the RF pulse, $B_{1}$, was calculated based on the given flip angle and the duration of the RF pulse.

\section{RF Pulse Shapes and Apodization}

All RF pulses are modulated in time, since they are finite in their time duration, which implies they also have a modified frequency distribution. This situation can be modeled mathematically by multiplying the ideal field $B_{1, \text { ideal }}(t)$ by a rect function which effectively represents the act of time truncation:

$$
B_{1}(t)=B_{1, \text { ideal }}(t) \cdot \operatorname{rect}\left(\frac{t}{\tau_{\mathrm{RF}}}\right)
$$

The rect function is defined as: 


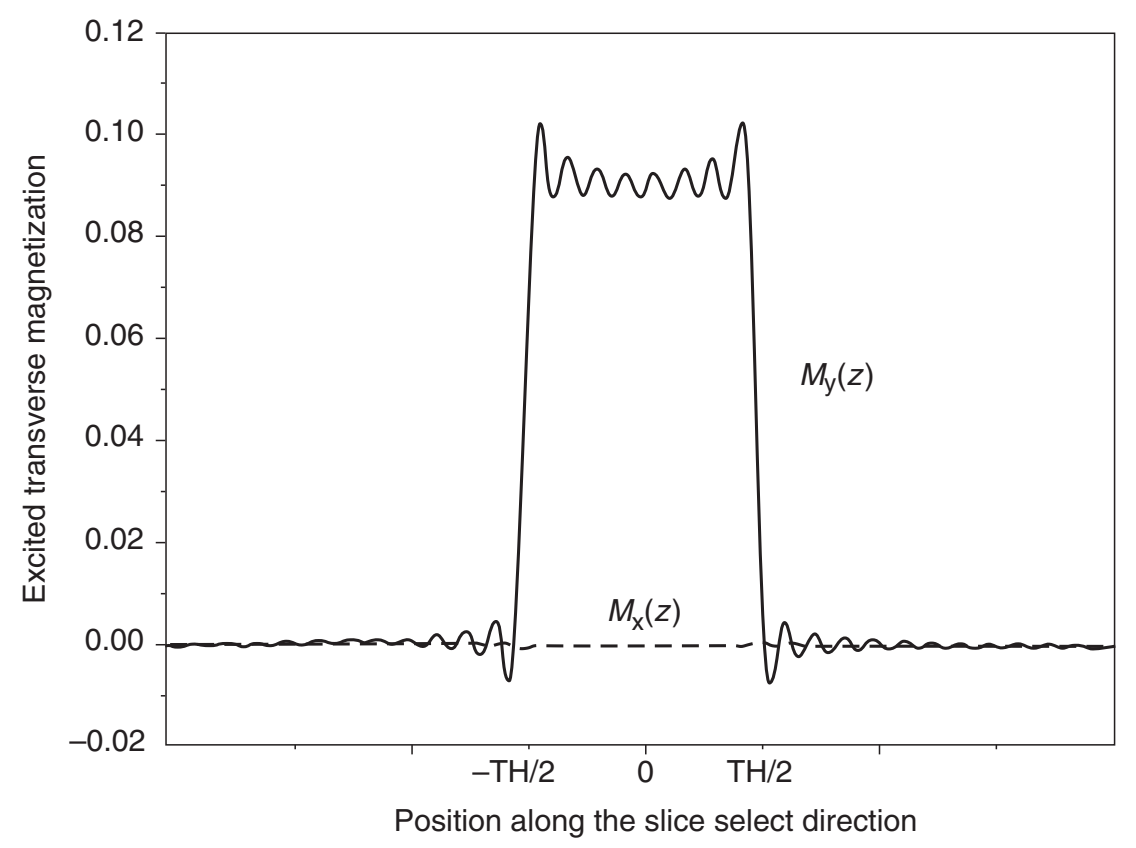

Figure B2.2.4 $\quad M_{x}(z)$ (dashed line) and $M_{y}(z)$ (solid line) are shown for a $5^{\circ}$ sinc pulse, as found by numerically integrating the Bloch equations. This result validates the low flip angle approximation that a $50 \%$ slice select refocusing gradient should give a maximal response for $M_{y}(z)$, with a negligible contribution from $M_{x}(z)$.

$$
\operatorname{rect}(u) \equiv \begin{cases}0 & u<-1 / 2 \\ 1 & -1 / 2 \leq u \leq 1 / 2 \\ 0 & u>1 / 2\end{cases}
$$

The inverse Fourier transformation of $B_{1}(t)$ leads to a frequency response (spatial response for the slice selection process) which is the convolution of the Fourier transformation of the ideal response with a sinc function (i.e., $\operatorname{sinc}(u) \equiv \sin u / u$, which is the Fourier transformation of a rect function):

$$
B_{1}(f)=B_{1, \text { ideal }}(f) *\left(\tau_{\mathrm{RF}} \operatorname{sinc}\left(\pi f \tau_{\mathrm{RF}}\right)\right)
$$

where the notation * represents convolution. We remind the reader that, strictly speaking, this inverse Fourier transform represents the low flip angle approximation. As the duration of the RF pulse increases, the function $\tau_{R F} \operatorname{sinc}\left(\pi f \tau_{\mathrm{RF}}\right)$ approaches a $\delta$-function and $B_{1}(f)$ approaches $B_{1, \text { ideal }}(f)$. Nevertheless, even in this limit, truncation artifacts will still exist, i.e., Gibbs ringing will occur near sharp boundaries.

In practice, an additional apodizing function is used to bring $B_{1 \text {,ideal }}(t)$ smoothly to zero and reduce truncation effects. A more general expression for the time dependence of the field is:

$$
B_{1}(t)=B_{1, \text { ideal }}(t) \cdot a(t) \cdot \operatorname{rect}\left(\frac{t}{\tau_{\mathrm{RF}}}\right)
$$

Radiofrequency Excitation and Reception

B2.2.7 

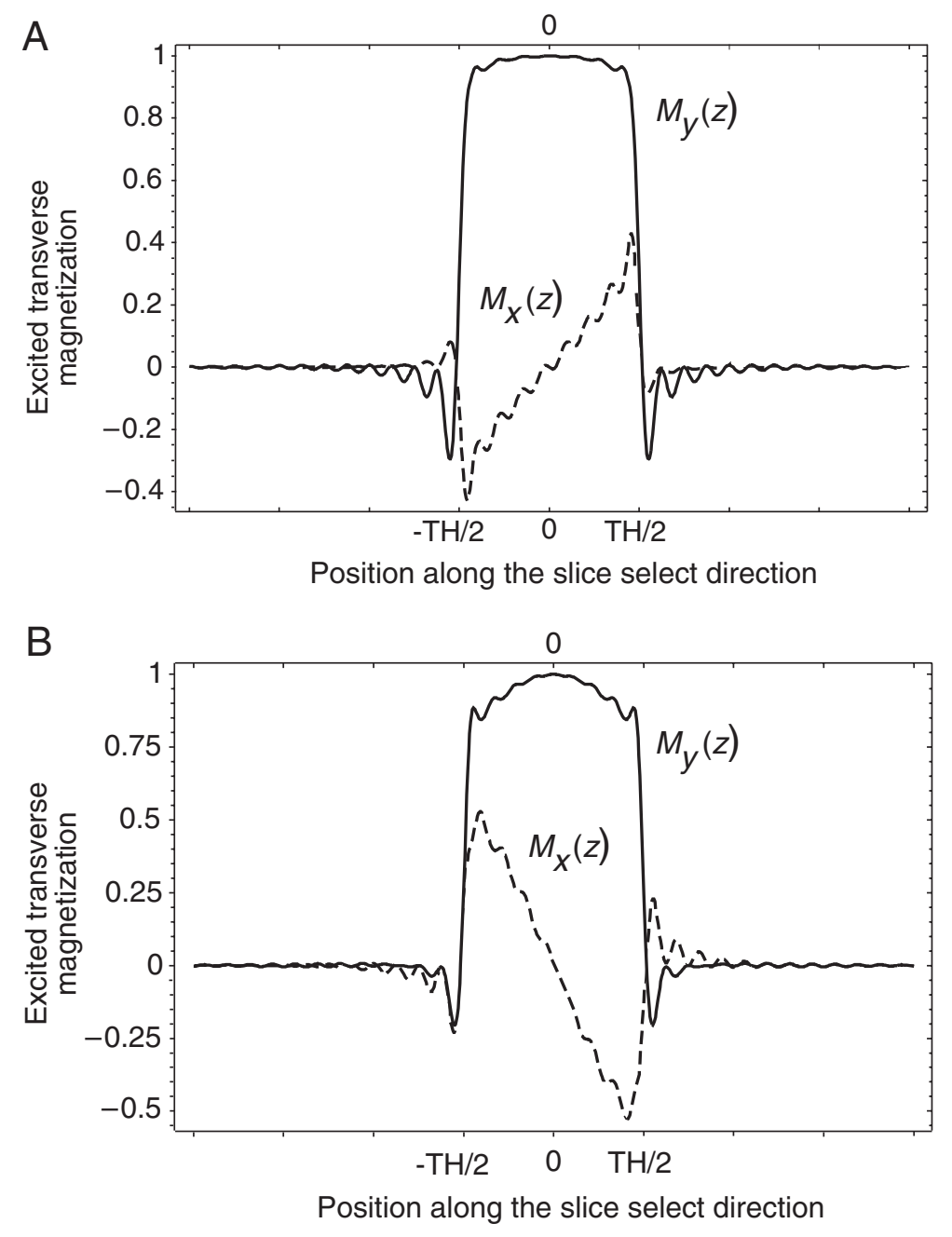

Figure B2.2.5 $M_{x}(z)$ and $M_{y}(z)$ are shown for a $90^{\circ}$ sinc pulse, as found by numerically integrating the Bloch equations. The area of the refocusing gradient pulse used in $(\mathbf{A})$ is $50 \%$ and in $(\mathbf{B})$ is $52 \%$. The $M_{x}(z)$ component can be minimized if the refocusing is chosen to be $\sim 50.6 \%$.

where $a(t)$ is an apodizing function and the frequency response of the pulse becomes:

$$
B_{1}(f)=B_{1, \text { ideal }}(f) * A(f) *\left(\tau_{\mathrm{RF}} \operatorname{sinc}\left(\pi f \tau_{\mathrm{RF}}\right)\right)
$$

where $A(f)$ is the inverse Fourier transformation of $a(t)$. The convolution theorem can be used to investigate the actual frequency content of the pulse.

An example of the effects of truncation and apodization on an ideal field profile as a function of time and frequency are shown in Figure B2.2.6. In Figure B2.2.6A, a truncated sinc function is shown, and its associated flip angle profile, $\theta(z)$, is shown in Figure B2.2.6B. (The small angle approximation can be obtained from Equation B2.2.3 and Equation B2.2.9.) Figure B2.2.6C shows the same sinc function after apodization with a Hanning filter. The excitation profile shown in Figure B2.2.6D demonstrates the smoothing effect which apodization introduces. It is important to reduce the ripples extending past the slice of interest as they either contribute signal from outside the slice of interest 
A

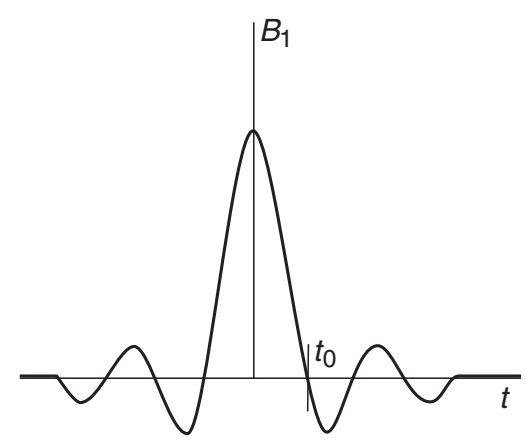

C

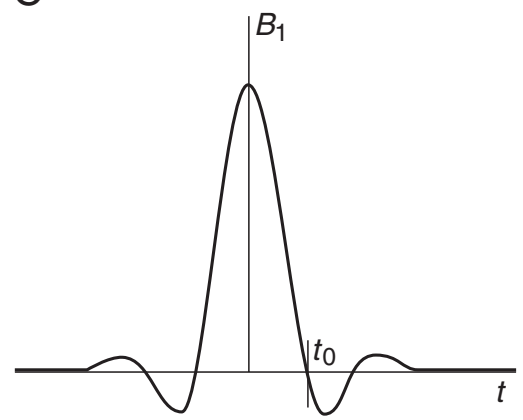

B
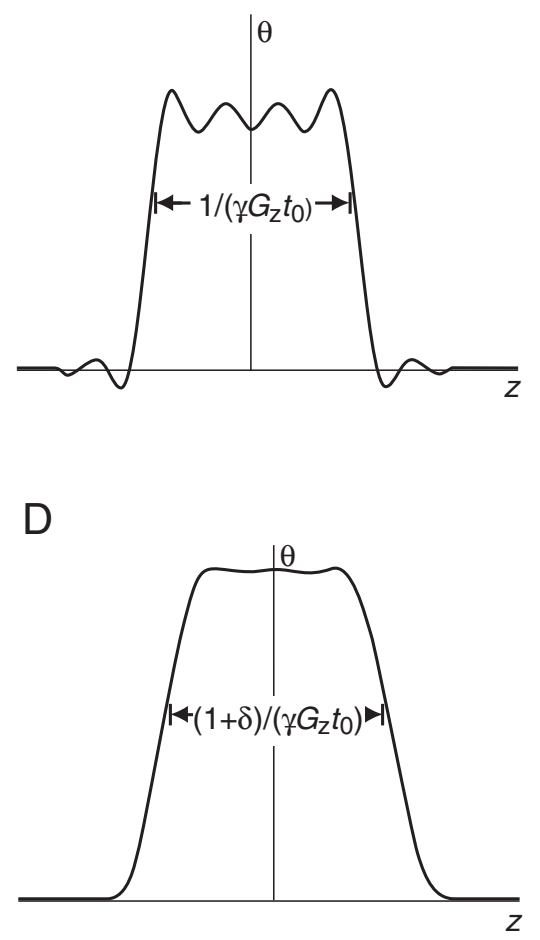

Figure B2.2.6 (A) A sinc pulse truncated by a rect filter and (B) its corresponding flip angle profile, which is contaminated by Gibbs ringing. (C) A smoothly truncated or apodized sinc function (a Hanning filter has been used), and its resulting $\theta$ response (D) is spatially smoother than that shown in $\mathrm{B}$. The differences between $\mathrm{B}$ and $\mathrm{D}$ demonstrate how smooth truncation of an RF pulse with an apodizing function can lead to a reduction of Gibbs ringing. The filtered profile is also broader than the original by the fraction $\delta$ as shown in $D$.

for 2-D imaging or alias into the images in 3-D imaging. Filtering the time domain input limits the spatial extent of the excited spins and the excitation profile, but increases the FWHM (full width at half maximum) relative to the original profile width.

\section{Hard and soft pulses}

A terminology has developed differentiating between pulses which are spectrally selective and those which excite a large range of frequencies. Short duration, high-amplitude pulses generally truncated with a rect function are referred to as "hard" pulses, since they are approximately an impulse in the time domain. However, their short duration in the time domain leads to a broad excitation profile in the frequency domain. For this reason, hard pulses are also referred to as nonselective pulses. They are generally used for saturation in the absence of gradients, or, for spectroscopy where the goal is to excite a large number of frequencies.

Pulses which are lower in amplitude and longer in duration may be used to select a narrow range of frequencies. Pulses which fit this description are often referred to as "soft" pulses. The RF pulses employed during slice selection are examples of soft pulses.

Radiofrequency Excitation and Reception

B2.2.9

Supplement 12 


\section{$\theta_{x, y}$-pulses}

Often, the direction along which $B_{1}$ points in the rotating reference frame during the application of the RF pulse is assumed to be irrelevant. However, there are applications where the relative phase of the applied $B_{1}$ field (i.e., the direction of the applied RF field) is important to the result. In these cases, a convention must be used to describe the axis along which $B_{1}$ is applied. Usually, $\theta_{x}\left(\theta_{y}\right)$ represents the flip angle where the RF pulse has been applied along the $x(y)$ axis in the rotating frame.

\section{Spatially Varying RF Excitation}

It is possible to excite an arbitrary 3-D region of space. The mathematical details of these excitations, however, can be somewhat laborious. An example of how conventional slice select pulses may be used to excite a rectangular region of space is introduced first. The notion of varying the time dependence of the gradients while the $B_{1}$ field is being applied is briefly discussed. Although usually a gradient along one of the Cartesian axes is used for defining the slice select direction, in general, multiple gradients can be applied to generate oblique slices.

\section{Two-dimensional "beam" excitation}

It is possible to excite a "beam" of spins spatially with a spin echo, instead of a slice. This is useful to avoid aliasing when a small FOV in a large object is desired. A sequence diagram for implementing a "beam" excitation is shown in Figure B2.2.7A. The first RF pulse and slice select gradient (in the $z$ direction) excite a slice in the conventional manner. During the $\pi$ pulse, a gradient lobe is placed (in the $y$ direction) perpendicular to the original slice select axis. Therefore, the $\pi$ pulse rotates spins in a slice perpendicular to the original slice. Only the rectangular set of spins which have experienced both pulses, as shown in Figure B2.2.7B, will form a spin echo. The gradients and bandwidths associated with the $\pi / 2$ and $\pi$-pulses determine the corresponding slice thickness in each direction for the phase encoding or partition encoding steps (see Fig. B2.2.7). The thickness of the beam will be chosen according to the size of the object being imaged.

In general, the slice select gradient associated with the $\pi$ pulse is placed along the phase-encoding direction to reduce the number of phase encoding steps and thus the total imaging time (for 2-D imaging, $T_{T}=L_{y} T_{R} / \Delta y$ ) while maintaining or improving resolution. The positioning and shape of the read gradient lobes are also important when employing this method. The slice-selective $\pi$ pulse acts on all spins in the $x$-z plane. Although the $\pi$-pulse may be very well designed, there will be some of these spins that undergo only a $\pi / 2$-rotation. They can contribute to the MRI signal. In order to minimize their effect on the image, additional gradients are added to the dephase and rephase gradient lobes (shown as shaded areas in Fig. B2.2.7). The gradient echo still occurs at $T_{\mathrm{E}}$ where the gradient areas are balanced, but the additional area before data collection dephases spins tipped from the $z$ axis into the transverse plane by the $\pi$ pulse. This additional gradient should be chosen to sufficiently dephase the unwanted magnetization before data are collected in a manner similar to that described in UNIT B2.3 for eliminating unwanted magnetization prior to slice selection. The difference is that, in this case, the goal is to ensure that the unwanted magnetization is dephased across a pixel width along the read direction at the echo, and not along the slice select direction. If the times separating the pulses and gradients shown in Figure B2.2.7 are reduced to zero, a single composite pulse of gradients and RF designed to excite a volume emerges.

Other configurations of the gradients are possible as long as they take into account the spins excited by the $\pi$ pulse and minimize their effect on the image. For example, one can utilize a similar method to image a voxel in MR spectroscopy. This approach is even more valuable when 3-D imaging must be done, but a limited field-of-view is desired.

Radiofrequency Excitation

B2.2.10 
A
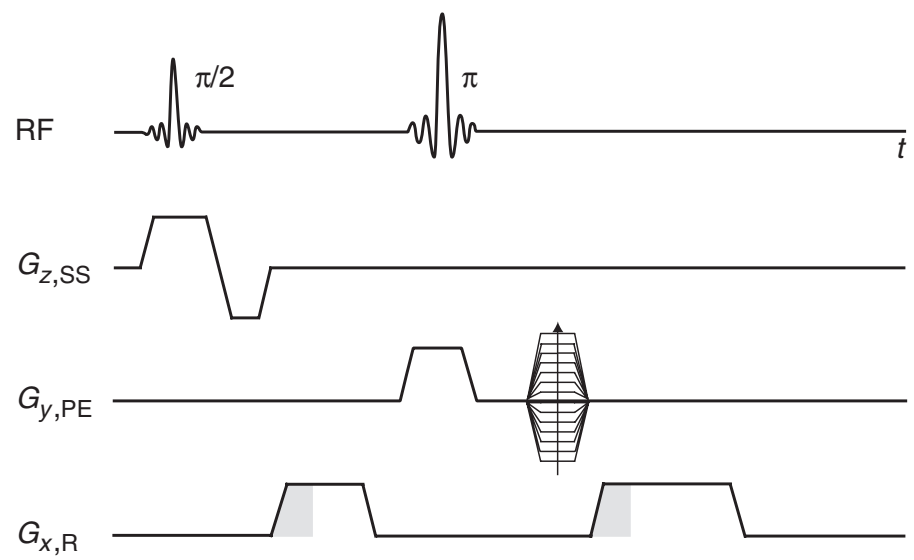

B

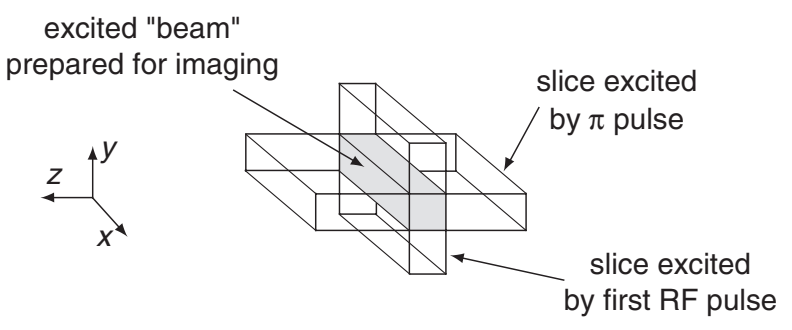

Figure B2.2.7 Sequence diagram (A) and schematic of the resulting excitation (B) when a rectangular beam of spins is excited. The number of phase encoding lines needed for the same resolution can be reduced by exciting smaller fields-of-view in two directions. This is achieved for a spin echo sequence, for example, by exciting a plane in one direction during the $\pi / 2$-pulse and by exciting a plane perpendicular to the first plane during the $\pi$-pulse. In this example, slice selection takes place along $z$ for the $\pi / 2$-pulse and along $y$ for the $\pi$ pulse. The dephasing and rephasing portions of the read gradient are usually lengthened (see shaded area along $G_{x, R}$ ) to allow "crushing" or dephasing of any transverse magnetization which may be created by the $\pi$-pulse.

\section{Time varying gradients and slice selection}

It can be seen from the previous arguments that the spatial region of excitation will be altered if the applied gradient varies during the slice select process. Low-flip-angle imaging is of particular interest in fast imaging applications and reducing the slice select time is often desirable. In these cases, it would be optimal to design the RF and gradient pulse so that the RF is on throughout the application of the gradient, including ramp times. Alternately, with extremely powerful gradients, a trapezoidal gradient may be exchanged for a triangular waveform.

\section{KEY REFERENCE}

Haacke, E.M., Brown, R.W., Thompson, M.R., and Venkatesan, R. 1999. Magnetic Resonance Imaging: Physical Principles and Sequence Design. John Wiley \& Sons, New York.

This text covers the technical aspects presented here, but in more detail, and also discusses more advanced materials.

Contributed by Yu-Chung Norman Cheng and E. Mark Haacke

Case Western Reserve University

Cleveland, Ohio

and The MRI Institute for Biomedical Research

Detroit, Michigan

Radiofrequency

Excitation and

Reception

B2.2.11 